\title{
A Technique for Measuring Atmospheric Diffusion ${ }^{1,2,3}$
}

\author{
Eugene W. Bierly and Gerald C. Gill \\ The University of Michigan
}

(Manuscript received 29 July 1962)

\begin{abstract}
A technique for measuring atmospheric diffusion with a "floating grid" system is described. Tracer sampling is accomplished using one or two airplanes flying arcs of circles at selected radial distances from the release point and flying selected levels on each arc. The planes are guided by a ground based radar. The centerline of the "floating grid" sampling network is aligned in the direction toward which the mean wind is blowing and can change during an experiment. Instrumentation necessary for the dispensing, sampling and analysis of the tracer material is described as well as the associated meteorological instrumentation. A brief description of the analysis of data using the "floating grid" system is also presented.
\end{abstract}

\section{Introduction}

There is a definite need for improvement in sampling networks. The usual fixed grid system with samplers on parallel arcs at fixed distances from the point of release does not give results that may be termed truly operational, but rather it gives results that are special cases of diffusion. Samples obtained from the fixed grid system are generally in a horizontal plane so it is difficult to make any quantitative statements about vertical diffusion when using this system. There is a need for a practical, operational type of diffusion study that will enable a user to know what the dispersion patterns will be under most atmospheric conditions, especially the extreme condition of inversion. This information is necessary so that either manual controls may be incorporated into plant operating procedures, or so that automatic control mechanisms may be designed and built into the plant for operational usage.

The "floating grid" system is one possible answer to this need. In experiments where the "floating grid" system has been used, fluorescent particles are emitted continuously from an elevated point source. Samples are taken on arcs of circles at various heights above the surface. The centerline of the sampling network floats with the mean wind direction. This system is depicted in Fig. 1. The tracer material used with the system, dispensers, samplers, and the "floating grid" system itself are discussed along with the associated meteoro-

\footnotetext{
${ }^{1}$ Publication No. 66 of the Meteorological Laboratories, Department of Engineering Mechanics, The University of Michigan.

${ }^{2}$ Contribution No. 21 from the Great Lakes Research Division, Institute of Science and Technology, The University of Michigan.

${ }_{3}^{3}$ Research conducted under the sponsorship of The National Science Foundation Grant No. G-11404 and of The Power Reactor Development Co., Detroit, Mich.
}

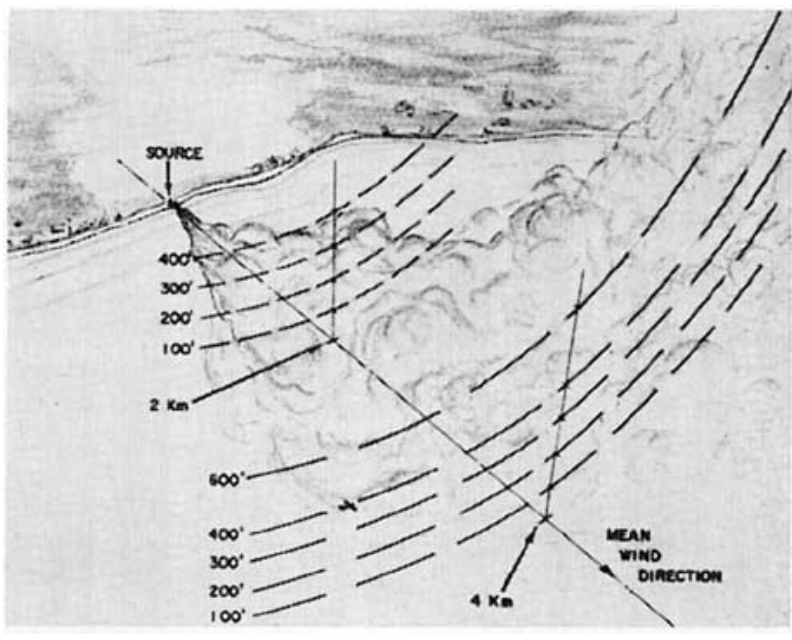

FIc. 1. Idealized view of "floating grid" sampling system.

logical equipment necessary for analysis of the data. A brief description of how the data are analyzed concludes the paper.

\section{Tracer material}

Several tracer materials were considered including oil fog smoke, sulphur dioxide, radioactive materials, uranine dye and fluorescent particles. From the above group, fluorescent particle material was chosen because it was a proven technique (Perkins et al., 1952; and Hilst, 1957), was non-hazardous to operators, was reasonably economical for reliable sampling at distances of $16 \mathrm{~km}$ or greater, and there was no absorption of the material by moisture in the air or by water surfaces.

The tracer material used is a mixture of zinc and cadmium sulfides known commercially as fluorescent paint pigment No. 2267. This pigment is manufactured 
by the U. S. Radium Corporation and is specially processed to a mean diameter of approximately $2.7 \mu$ for use as an atmospheric tracer. The calculated Stokes settling rate for a spherical particle of density 4 of this diameter is about $3 \mathrm{~m}$ per hr. The material contains an average of $2.3 \times 10^{10}$ detectable airborne particles per gram. Physical and chemical properties of the fluorescent particle material have been given by Leighton. ${ }^{4}$ When this material is placed under ultraviolet light, it fluoresces brilliantly with a yelloworange to green color thus enabling the particles to be counted either by eye under a microscope or by use of a scintillation type counter.

\section{Fluorescent particle system}

The fluorescent particle system consists of three major components--dispenser, sampler and counting equipment.

Two types of dispenser have been used with the "floating grid" system. Fig. 2 shows the earlier version which is a type developed by the Stanford Aerosol Laboratories and described in detail by Leighton. ${ }^{5}$ It consists of a high speed blower (1), hopper (2), stirrer (4), feed mechanism (5), and a power supply for the motors (6). The hopper holds approximately $2 \mathrm{~kg}$ of fluorescent particle material. The emission rate is

${ }^{4}$ Leighton, P. A., 1955. The Stanford fluorescent-particle tracer technique. An operational manual, Dept. of Chemistry, Stanford Univ., Palo Alto, Calif.

${ }_{5}$ Ibid.

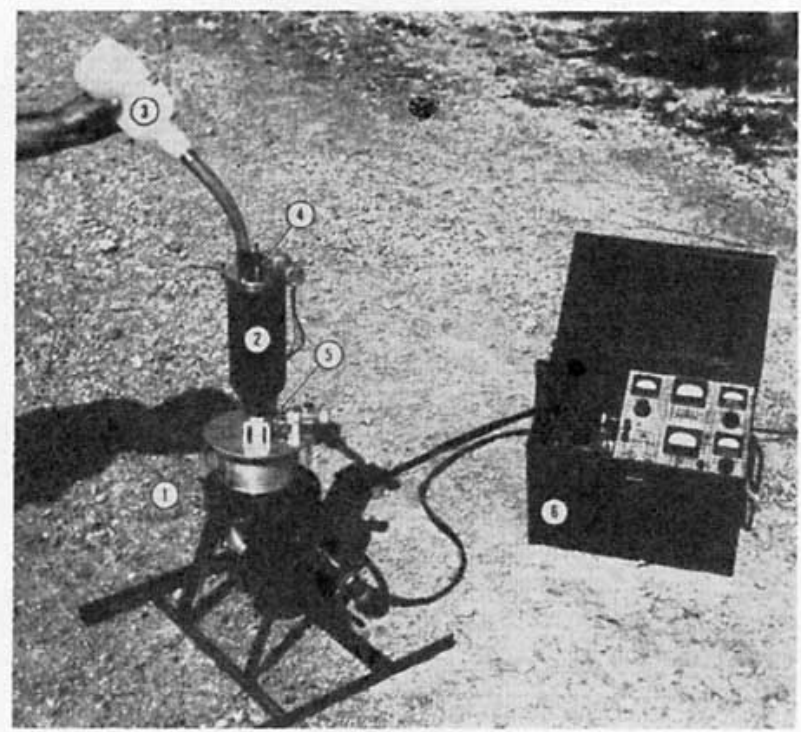

Fig. 2. Stanford aerosol generator and power supply for fluorescent particles: (1) Skil blower, (2) hopper, (3) polyethylene bottle for adding fluorescent particle material, (4) stirrer, (5) feed mechanism, (6) power supply.

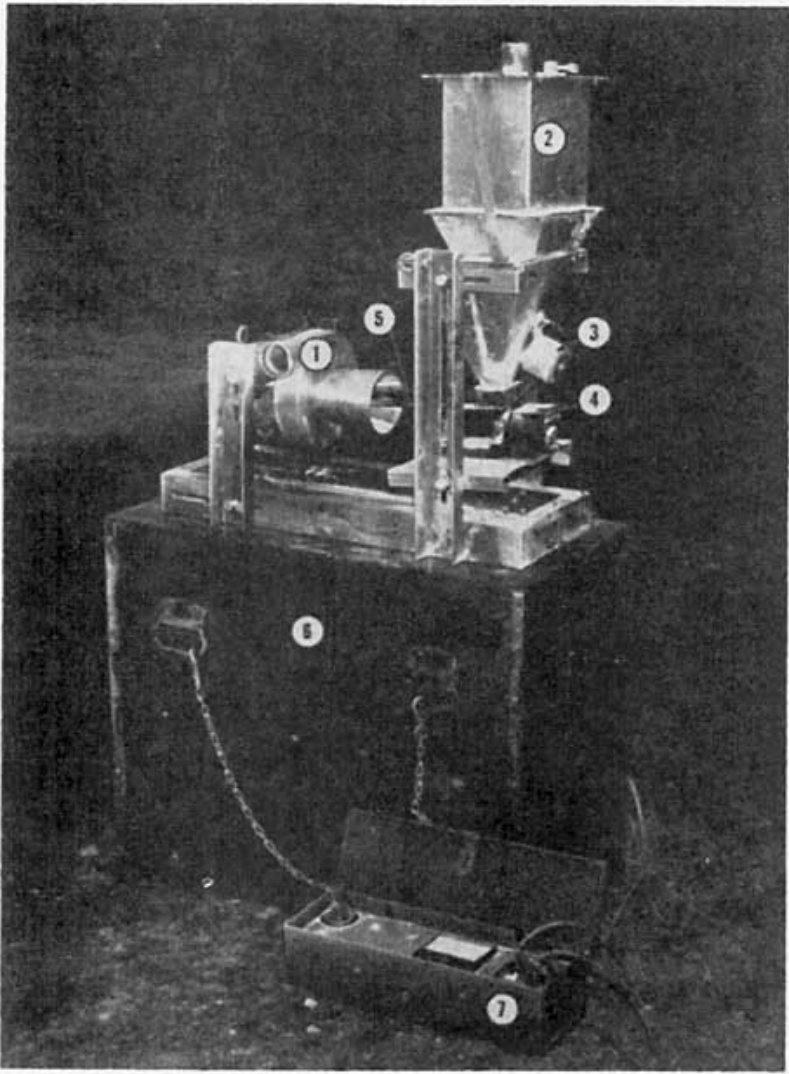

FiG. 3. University of Michigan aerosol generator and power supply for fluorescent particles: (1) Skil blower, (2) hopper, (3) hopper vibrator, (4) chute vibrator, (5) chute, (6) carrying case, (7) power supply and control panel.

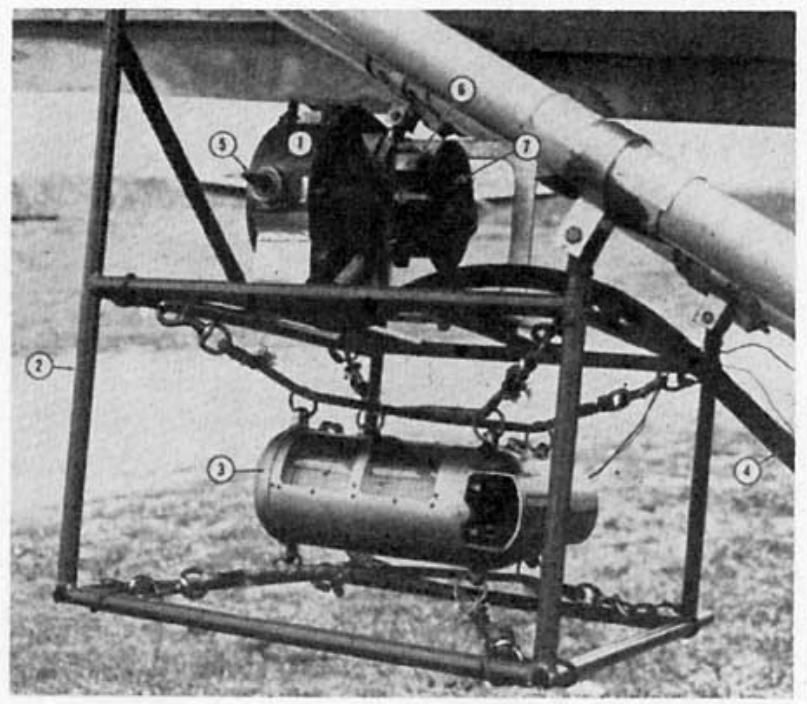

Fig. 4. Closeup of sampler and aerometeorograph: (1) sampler, (2) supporting frame, (3) aerometeorograph, (4) vacuum hose, (5) isokinetic cone, (6) rotary solenoid, (7) dial plate corresponding to sample number. 
adjustable from 30 to $75 \mathrm{gm}$ per min. Refilling of the hopper is carried out during operations by use of squeeze bottles (3) as shown in Fig. 2. The rate of emission is calculated by weighing the fluorescent particle material put into the dispenser prior to and during the experiment and then weighing the unused material at the end of the experiment. Laboratory tests indicate that the emission rate remains steady within \pm 10 per cent for operating periods of 1 to $4 \mathrm{hr} .^{6}$

Fig. 3 shows another type of dispenser developed at The University of Michigan Meteorological Laboratories. It was built to provide a much higher rate of emission of fluorescent particle material and a larger storage bin. Both qualities are necessary when running for long periods and when samples are taken at distances up to $32 \mathrm{~km}$ from the source. The dispenser operates by use of vibrators (3) and (4) which cause the fluorescent particle material to run from the hopper (2) down a closed, inclined chute (5) into a Skil blower (1) from which it is dispensed. As in the Stanford model, the fluorescent particle material is broken into individual particles by the blower before leaving the generator. The hopper is weighed before and after the operation, to measure the fluorescent particle material emitted during the experiment. For 20 -min test runs the minute to minute variation did not exceed \pm 3 per cent of the mean emission rate. Each setting of the vibrators is equivalent to a fixed output of fluorescent particle, the output range being from 12 to $250 \mathrm{gm}$ per min. The total capacity of the hopper is $18 \mathrm{~kg}$.

The sampling equipment consists of Stanford drum samplers mounted on an aircraft as shown in Fig. 4. Air is drawn through an isokinetic cone (5) and then into the sampler through a narrow horizontal slit. After passing through the slit the air turns sharply and passes out of the sampler through rubber tubing (4). Any fluorescent particle material accompanying the air drawn into the sampler is unable to make the rapid turn necessary to pass out of the sampler and impinges on the greased surface of aluminum foil that overlies the drum.

A flow rate of $50 \ell$ per min is maintained through the sampler at all times. Synchronous motor timers permit the observer to select fixed sampling periods of $7 \frac{1}{2}, 15$, 30,60 or $120 \mathrm{sec}$. A rotary solenoid inside the sampler housing advances the drum 3 deg each time the circuit is closed. Each drum has a potential of 120 samples but exposures do not usually exceed 110 samples. One plane is equipped with tandem samplers which double the number of samples that may be obtained before having to land for refueling or to change sampling drums.

The sampling drums are removed from the sampler upon landing and placed in airtight containers until they can be examined in the laboratory. The examina-

\footnotetext{
${ }^{6}$ Ibid.
}

tion of the drums and the counting of the fluorescent particles on the drum takes place in a photographic dark room well away from any possible source of fluorescent particle material. A 100-power microscope adapted so the drum can be rotated under the eyepiece is used for counting. Ultraviolet illumination is provided by a 100 watt General Electric model BH-4 mercury arc lamp fitted with a filter. The details of this technique as well as some of the problems involved in counting are described thoroughly by Leighton. ${ }^{7}$

\section{Floating grid system}

Airplanes are used as sampling platforms so measurements can be made over water as well as over land. Helicopters have been used successfully for gas sampling (Gartrell and Carpenter, 1955) but mounting an efficient particle sampler would be difficult because of turbulence caused by the rotor. The airplanes used are single engine, high wing monoplanes which can be operated at $90 \mathrm{mph}$ and still maintain stability and maneuverability. The planes can stay in the air for over three hours at one time.

During experiments using the "floating grid" system, the planes fly a circular path at radial distances of 2,4 , 8,16 and $32 \mathrm{~km}$ from the source. At each radial distance, various altitudes are flown depending upon specific weather conditions. Heights of $100,200,300$, $400,600,800$ and $1000 \mathrm{ft}$ are usually flown at 2 and $4 \mathrm{~km}$; while heights of $100,300,500,800,1000,1500$ and $2000 \mathrm{ft}$ are usually flown at 8 and $16 \mathrm{~km}$. Table 1

TABLE 1. Resumé of radial distances, sampling time and distances covered by the airborne sampling unit.

\begin{tabular}{cccc}
\hline \hline $\begin{array}{c}\text { Radial } \\
\text { distance } \\
(\mathrm{km})\end{array}$ & $\begin{array}{c}\text { Sampling } \\
\text { time } \\
(\mathrm{sec})\end{array}$ & $\begin{array}{c}\text { Distance covered } \\
(\mathrm{deg})\end{array}$ & $\begin{array}{c}\text { per sample } \\
(\mathrm{m})\end{array}$ \\
\hline 2 & 15 & 17.3 & 600 \\
4 & 30 & 17.3 & 1200 \\
8 & 60 & 17.3 & 2400 \\
16 & 120 & 17.3 & 4800 \\
32 & 120 & 8.7 & 4800 \\
\hline
\end{tabular}

gives a resumé of the sampling time in seconds and the distance covered in degrees and meters at the various radii.

As the sampler changes from one setting to another, there is a lag time of $0.2 \mathrm{sec}$ which is equivalent to traversing a distance of approximately $8 \mathrm{~m}$ at the aircraft's cruising speed of $90 \mathrm{mph}$.

The width of the sampling array is also varied according to the atmospheric conditions. It is never less than $90 \mathrm{deg}$ and averages $120 \mathrm{deg}$ in width. This width is determined by wind direction fluctuations at the

${ }^{7}$ Ibid. 
release site using a safety factor of 20-30 deg on each side of the extremes of the wind fluctuations so the plume will be completely sampled even under meandering conditions.

An airplane begins sampling at $100 \mathrm{ft}$ on the $2-\mathrm{km}$ arc. Constant altitude is maintained until the sampling is completed, then the plane gains altitude as it proceeds around in a circular path to the starting position. Such an operation insures that the plane is in a constantly banked position which enables the pilot to see both the source and landmarks. The spiral is continued until the highest altitude is sampled, then the plane proceeds to the next radial arc and begins sampling at the lowest level. Fig. 1 is an idealized picture of a plane sampling on the "floating grid" system. When using two planes simultaneously, the upper plane flies slightly behind the lower plane so the upper pilot can keep in line with the lower plane. The lower plane takes the lower half of the sampling network while the upper plane starts in the middle and samples the upper half.

The position of the planes in space is determined by a radar set located at the source. From the radar's PPI scope the radial distance from the source and the azimuth angle are obtained. The height of the plane above the surface is obtained from the plane's altimeter. This value is double checked by a surveying altimeter carried by the observer. The plane's altimeter is checked and set before each take-off in the standard manner. From these three values the plane's coordinates are known at any instant of time.

There are errors inherent in positioning the plane when using radar. The altitude of the plane is the most nearly correct measurement, usually being in error by less than $\pm 20 \mathrm{ft}$. The azimuth position when over a water surface can be in error by as much as $\pm 5 \mathrm{deg}$ and the radial distance can be in error by \pm 5 per cent. Over land where the pilot can use landmarks, the azimuth error is $\pm 2 \mathrm{deg}$ and the error in radial distance is \pm 2 per cent. The magnitude of these errors over a water surface is great enough to cause a truly normal distribution of particles to appear as a non-normal distribution or vice versa.

The radar in general serves as a guide to the pilots. When sampling over a water surface, the pilots start their banked turns over the short line while the radar operator helps them correct their radial distance. The radar operator tells the observer when to start and when to stop sampling.

Positive identification of the sampling aircraft on the PPI scope is provided by placing a transponder in the cargo section of the plane. As the radar beam passes over the plane, the transponder is triggered and sends out a strong signal which appears on the PPI scope.

Radio communication is maintained between the plane observers and pilots and the radar operator. All communications are recorded at the radar site on a continuously operating magnetic tape. The tape provides an excellent time log of all events reported during a run. When sampling, the plane's observers give the sample numbers as they change while the radar operator calls out the bearing of the plane every $10 \mathrm{deg}$. Later analysis then enables the correlation of the sample number and its particle count with its position in space.

The "floating grid" technique has the advantage that samples may be taken under wind conditions from any direction. Even though the mean wind direction changes, the data collected can still be analyzed unless a significant wind shift occurs while sampling at a given altitude and at a given radial distance. If a wind shift of greater than 45 deg occurs, then the entire arc is resampled. Although the first samples are taken on the arc closest to the source, there is no reason why the technique cannot be started on the arc farthest from the source. Sampling using the "floating grid" system may proceed under all except the worst atmospheric conditions. Samples are not taken in the rain because of the probable washout of the fluorescent particle material by the droplets. FAA regulations make it mandatory that Visual Flight Rules be adhered to so there can be no sampling when clouds are below $1000 \mathrm{ft}$ and visibility less than $3 \mathrm{mi}$. In some areas night flights would be possible; however, in areas of high air traffic density, flights must be restricted to day-time hours.

Even with the above mentioned restrictions the "floating grid" system has been found to be effective in obtaining diffusion measurements successfully.

\section{Associated meteorological measurements}

In the specific experiments conducted at the Enrico Fermi Atomic Power Plant using the "floating grid" system, a 100-ft meteorological tower was located on the shore line as illustrated in Fig. 5. Three aerovanes were placed at 24, 56 and $102 \mathrm{ft}$ for continuous measurement of wind speed and wind direction. Two GelmanGill bivanes for measuring wind fluctuations in the horizontal and vertical planes were located at 24 and $56 \mathrm{ft}$. Aspirated shielded thermocouples were at 25, 56, and $100 \mathrm{ft}$ on the tower and in a thermoscreen at $4 \mathrm{ft}$. Temperature differences between $4 \mathrm{ft}$ and the other levels were recorded continuously on a Bristol recorder using a cycling time of $6 \mathrm{~min}$. Fig. 5 shows a smoke generator in operation which was used to guide pilot and observer. At the Fermi site the fluorescent particle material was released from a dispenser at the 56- $\mathrm{ft}$ level as illustrated by (5) of Fig. 5. Fig. 4 shows the meteorological instrumentation which was mounted on the wing of the plane. It consisted of a Friez aerometeorograph mounted in such a manner that vibration was reduced to a minimum. The aerometeorograph recorded temperature, pressure and humidity from the time of the take off until landing. A time marker pro- 


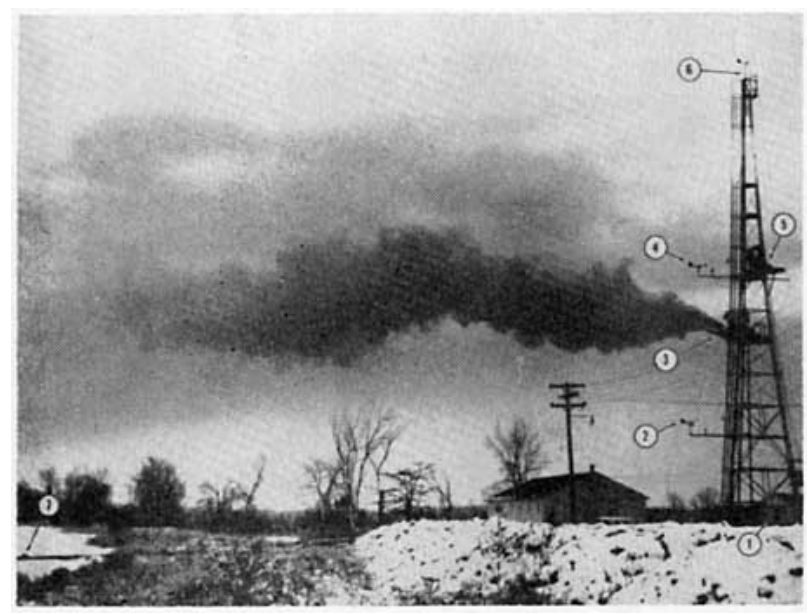

Fig. 5. View of meteorological installation looking south: (1) communications shack, (2) $25 \mathrm{ft}$ aerovane and bivane, (3) oil-fog generator and radar antenna at $45 \mathrm{ft}$ level, (4) $56 \mathrm{ft}$ aerovane and bivane, (5) fuorescent particle dispenser, (6) $102 \mathrm{ft}$ aerovane and radio antennae, (7) edge of Lake Erie.

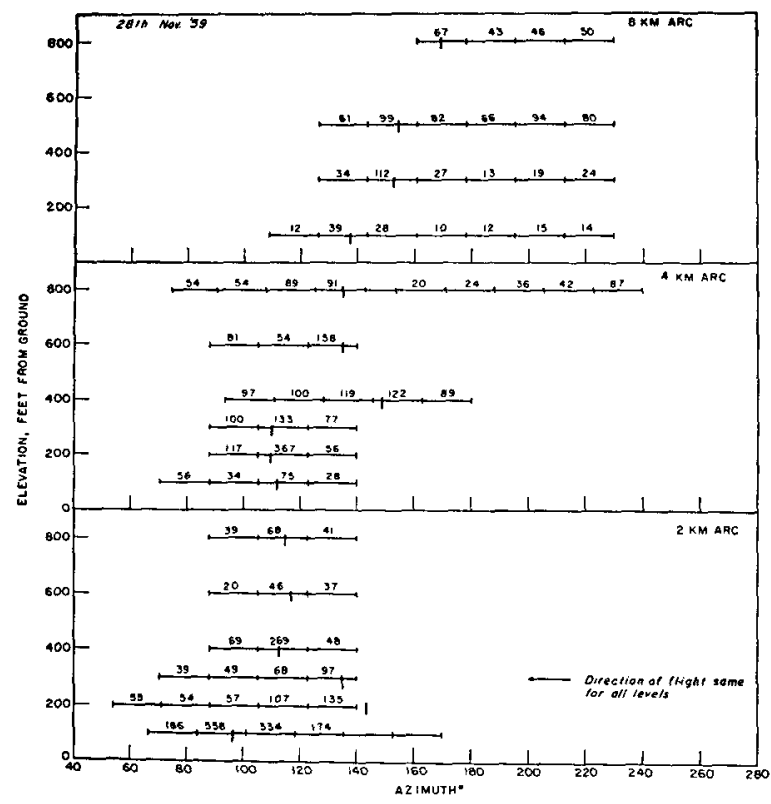

Fig. 6. Raw counts of fluorescent particle material from 28 November 1959 plotted according to azimuth and height above the lake surface. Vertical dashes show the estimated centerline of the plume.

vided a tick so that significant points could later be identified. A shielded wet and dry thermocouple which records automatically inside the plane has now replaced the aerometeorograph.

\section{Analysis of data}

The fluorescent particle counts from the drums can be converted to concentrations. Plots similar to Fig. 6 are made which show the particle count over the angular distance traversed during each sample.

The objective of the analysis is to compute values of Sutton's $C_{y}$ and $C_{z}$ using an assumed value of $n$. The details of this procedure are discussed by Bierly and Hewson (1963). These data are handled in two separate ways, both of which give values of $C_{y}$ and $C_{z}$. The first method is purely mathematical. Using an assumed value of $n$ arrived at by analysis of the airplane soundings, the value of $C_{z}$ is computed using the ratio of the crosswind concentrations at two adjacent levels on the same radial distance. The value of $C_{y}$ is then computed using the formula for the centerline concentration at any point downwind from a continuous point source. The second method is graphical and assumes that the fluorescent particle material is distributed normally in space. The value of $C_{y}$ is obtained from a plot on normal probability paper of the cumulative relative percentage frequency of the fluorescent particle material on any arc at any given height. The standard deviation of the count is obtained and this is changed into $C_{y}$ using Sutton's formula (Sutton, 1953).

$$
C=\sqrt{2} \sigma / x^{(2-n) / 2}
$$

where

$C=$ generalized coefficient of diffusion

$\sigma=$ standard deviation of the concentration or count $x=$ horizontal distance downwind from the source

$n=$ nondimensional number between 0 and 1 related to the diffusing power of the atmosphere.

A similar technique is used to obtain $C_{z}$ except that the maximum concentration on each arc for a given radial distance is plotted on the normal probability paper.

Both methods give results which are compatible with values obtained from other similar experiments. Furthermore the results from the two separate methods give values which are compatible with each other.

\section{Conclusions}

The "floating grid" system provides a practical method for measuring diffusion under various atmospheric conditions. It gives diffusion data in both the horizontal and vertical planes. Although there are small errors inherent in the system, especially in positioning the plane, the system does enable diffusion studies to be made on an operational basis.

This system has been used successfully in obtaining diffusion measurements near a shoreline where atmospheric turbulent regimes are complex. It is possible to use the "floating grid" system to determine diffusion potential of the atmosphere in order to assess the desirability of a plant location at a particular site or to assess the effect of an already established industrial plant on its environs. 


\section{REFERENCES}

Bierly, E. W., and E. W. Hewson, 1963: Atmospheric diffusion studies near a lake shore. J. appl. Meleor., 2, in press.

Gartrell, F. E., and S. B. Carpenter, 1955: Aerial sampling by helicopter : a method for study of diffusion patterns. J. Meteor., 12, 215-219.
Hilst, G. R., 1957: The dispersion of stack gases in stable atmospheres. J. air poll. cont. Assoc., 7, 205-210.

Perkins, W. A., P. A. Leighton, S. W. Grinnell and F. X. Webster, 1952: A fluorescent atmospheric tracer technique for mesometeorological research. Proc. 2nd National Air Pollution Symposium, Pasadena, Calif.

Sutton, O. G., 1953: Micrometeorology. New York, McGraw-Hill, $286 \mathrm{pp}$. 